The author sincere thanks gratitude to Academician of the National Academy of Sciences of Ukraine professor B.T. Stegniy (NSC IECVM NAAS of Ukraine) and the $\mathrm{PhD}$ of biological sciences O. V. Bilynska (V. Ya. Yuriev Research Institute of NAAS of Ukraine)

\title{
References:
}

1. Chen Y, Liang W, Yang S, Wu N, Gao H,Sheng J, Yao H, Wo J, Fang Q, Cui D, Li Y, Yao X, Zhang Y, Wu H,Zheng S, Diao H, Xia S, Zhang Y, Chan KH, Tsoi HW, Teng JL, Song W, Wang P, Lau SY, Zheng M, Chan JF, ToKK, Chen H, Li L, Yuen KY. 2013. Human infections with the emerging avian influenza A H7N9 virus from wet market poultry: clinical analysis and characterisation of viral genome. Lancet 381:1916-1925. doi: 10.1016/S0140-6736(13)60903-4.

2. Neumann G., Macken C.A., Kawaoka Y. 2014. Identification of amino acid changes that may have been critical for the genesis of A(H7N9) influenza viruses. J Virol 88:4877-4896. doi: 10.1128/JVI.00107-14.

3. Kageyama T., Fujisaki S., Takashita E., Xu H., Yamada S., Uchida Y., Neumann G., Saito T., Kawaoka Y., Tashiro M. 2013. Genetic analysis of novel avian A(H7N9) influenza viruses isolated from patients in China, February to April 2013. Euro Surveill 18:20453.

4. Zhang Q., Shi J., Deng G., Guo J., Zeng X., He X., Kong H., Gu C., Li X., Liu J., Wang G, Chen Y, Liu L, Liang L, LiY, Fan J, Wang J, Li W, Guan L, Li Q, Yang H, Chen P, Jiang L, Guan Y, Xin X, Jiang Y, Tian G, Wang X, Qiao C,Li C, Bu Z, Chen H. 2013. H7N9 influenza viruses are transmissible in ferrets by respiratory droplet. Science 341:410-414. doi: 10.1126/science.1240532.

5. Liu, C.Y., Ai J.H. [Virological characteristics of avian influenza A H7N9 virus]. Zhongguo Dang Dai Er Ke Za Zhi 2013; 15: 405-408. 2013/06/26.

6. Mei Z., Lu S., Wu X. Avian influenza A(H7N9) virus infections, Shanghai, China. Emerg Infect Dis 2013; 19: 1179-1181. 2013/06/19. doi: 10.3201/eid1907.130523.

7. Zhou L., Tan, Y., Kang, M. Preliminary epidemiology of human infections with highly pathogenic avian influenza A(H7N9) virus, China, 2017. Emerg Infect Dis 2017; 23: 1355-1359. 2017/06/06. doi: 10.3201/eid2308.170640.

8. Wang, D., Yang, L., Zhu, W. Two outbreak sources of influenza A (H7N9) viruses have been established in China. J Virol 2016; 90: 5561-5573. doi: 10.1128/JVI.03173-15.

\section{THE INTENSITY OF INFLAMMATION IN THE PULMONARY SYSTEM OF RATS IN RESPONSE TO THE INHALATION EFFECT OF NITROGEN(IV) OXIDE}

\section{Vitalii Ivchuk $^{1}$}

DOI: https://doi.org/10.30525/978-9934-588-11-2_2

A pathological inflammatory response in response to exposure to industrial airborne pollutants is the basis for the occurrence of chronic obstructive pulmonary disease (COPD) of occupational etiology [1, p. 995; 2, p. 1207]. Industrial risk factors that can cause this pathology are particles of industrial dust and aggressive gases that are in the air of the working area. The latter, among workers in the mining

\footnotetext{
${ }^{1}$ Ukrainian Scientific Research Institute of Industrial Medicine, Ukraine
} 
and metallurgical sectors of heavy industry, can initiate the development of inflammation of the respiratory tract. Clinical observations have shown that even in the post-contact period, with COPD, inflammation in the bronchopulmonary apparatus persists [3, p. 146; 4, p. 124]. The presence of numerous scientific publications leaves insufficiently studied many aspects of the pathogenesis of COPD. In particular, questions regarding the selectivity of the development of this professional pathology, the lack of inhibition of inflammation reactions in the postcontact period remain insufficiently covered in the literature. The discussion on the causes of exacerbations of COPD of professional etiology remains open $[1$, p. 995; 3 , p. 151]. Since the cells of innate and adaptive immunity, vascular endothelium and bronchopulmonary epithelium are involved in the course of chronic inflammation reactions, a more detailed study of the immune mechanisms of the onset and development of COPD of professional etiology will slightly open the veil of the pathogenesis of this disease.

The aim of the study was to assess changes in the intensity of inflammation reactions in the pulmonary system of experimental animals upon inhalation of nitrogen(IV) oxide.

Studies were performed on male Wistar rats $(n=35)$ weighing $160-180 \mathrm{~g}$. Rats were divided into 5 groups: Group $1-$ control $(\mathrm{n}=7)$; Group $2-\mathrm{NO}_{2}$ exposure for 15 days $(\mathrm{n}=7)$; Group $3-\mathrm{NO}_{2}$ exposure for 30 days $(\mathrm{n}=7)$; Group $4-\mathrm{NO}_{2}$ exposure for 60 days $(n=7)$; Group 5 - recovery period for 6 months $(n=7)$. The animals were kept on a standard diet. A model of the stage-by-stage formation of COPD, from an acute reaction to damage to a chronic process, was reproduced using the inhaled exposure to nitrogen(IV) oxide [5, p. 8]. Animals were removed from the experiment by cervical dislocation. All manipulations with experimental animals were carried out following the rules of the European Convention for the Protection of Vertebrate Animals used for research and other scientific purposes, as well as in accordance with scientific and practical recommendations for the maintenance and handling of laboratory animals. Bronchoalveolar lavage was performed on isolated lungs with isotonic sodium chloride solution at room temperature. Using a hemocytometer in $1.0 \mathrm{ml}$ of bronchoalveolar lavage fluid, the total cell count was calculated and differential cell analysis was performed. Functional activity of alveolar macrophages was determined using standard latex particles. By the method of selective precipitation with polyethylene glycol in the blood serum, the content of circulating immune complexes was determined. By the method of enzyme-linked immunosorbent assay in blood serum and bronchoalveolar lavage fluid, the concentrations of tumor necrosis factor alpha $(\mathrm{TNF} \alpha)$ and transforming growth factor beta (TGF $\beta$ ) were determined. Statistical processing of experimental data was carried out using STATISTICA 6.0 software (StatSoft, USA). The significance of differences in values between independent quantitative values was determined using Student's ttest. Changes were considered statistically significant at $p<0.05$.

Significant changes in the cellular composition of bronchoalveolar lavage fluid were observed on day 15 of $\mathrm{NO}_{2}$ inhalation. So, in comparison with the control, the number of macrophages decreased by 2.4 times, while the content of neutrophils and 
lymphocytes increased by 9.5 and 4.8 times, respectively. The number of lymphocytes was consistently high throughout the entire time period of the experiment. After 30 days of $\mathrm{NO}_{2}$ exposure, the number of neutrophils approached control values. Until the completion of the recovery period, it was stable. At the same time, after 60 days of inhalation of $\mathrm{NO}_{2}$, the number of macrophages approached the indices of the control group of animals.

A study of the phagocytic activity of alveolar macrophages showed no statistically significant changes. However, it can be noted that there is a relationship between the gradual increase in the phagocytic number and phagocytic index and the duration of exposure to $\mathrm{NO}_{2}$ - its strength increases with time.

The most significant changes in the concentration of circulating immune complexes were observed after 60 days of experience. There was 9.2 and 3.8 times increase in the content of circulating immune complexes with high molecular weights and average molecular weights, respectively. A mutually opposite trend between the concentration of circulating immune complexes of various molecular weights was observed after the completion of the recovery period of experimental animals. So, in the blood serum the content of circulating immune complexes with high molecular weight decreased by almost 4.5 times. The concentration of circulating immune complexes with an average molecular weight remained almost at the level of the animal group after 60 days of $\mathrm{NO}_{2}$ exposure and exceeded the control group by more than 3.5 times. Since circulating immune complexes with an average molecular weight settle in the lung tissue and thereby contribute to the launch of the cascade of release of cytokines and chemokines, it becomes obvious that these complexes exhibit the greatest pathogenic effect. In addition, it is known that an obstacle in the development of repair processes in lung tissue is an increase in the content of these immune complexes, which, thereby, induce the persistence of inflammation.

The concentration of TNF $\alpha$ in blood serum and bronchoalveolar lavage fluid increased in proportion to the time period of the effect of $\mathrm{NO}_{2}$ in experimental animals. In blood serum, it increased by 3.2 times, and in bronchoalveolar lavage fluid by almost 145.0 times. The completion of the recovery period of the experimental animals led to a decrease in the level of TNF $\alpha$ in the blood serum by 2.9 times, which brought it almost to the level of the control group. While the concentration of this factor in bronchoalveolar lavage fluid remained almost at the level of numerical values of the group of animals after 60 days of $\mathrm{NO}_{2}$ exposure, which indicated the continuation of reactions in the focus of inflammation.

A similar dynamic was also revealed in the analysis of the content of TGF $\beta$ in blood serum and bronchoalveolar lavage fluid of rats of experimental groups. The effect on rats of $\mathrm{NO}_{2}$ for 30 days showed an increase in the concentration of TGF $\beta$ in blood serum by 2.6 times, in bronchoalveolar lavage fluid by 3.2 times compared with the group of animals, where the exposure lasted 15 days. The rapid growth of this factor by 33.0 and 14.7 times, respectively, in blood serum and bronchoalveolar lavage fluid was observed after 60 days of exposure to $\mathrm{NO}_{2}$. After completion of the recovery period, the serum TGF $\beta$ content decreased by 14.2 times compared with the group of animals where the exposure lasted 60 days, while in the bronchoalveolar 
lavage fluid almost did not change. It is known that TGF $\beta$ plays the role of one of the leading regulators of the processes of fibrosis and elastolysis.

The results of experimental studies indicate the presence of persistence of the chronic inflammatory process in the bronchopulmonary system of rats, which occurs as a result of prolonged exposure to $\mathrm{NO}_{2}$. With the occurrence of such an inflammatory reaction, obviously, there is the possibility of the formation of an autoimmune mechanism to support the process. The occurrence of morphological changes in the lung tissue may be due to the presence of an excess concentration of circulating immune complexes. After all, it is known that the latter are involved in the development of immunopathological reactions and thereby contribute to lengthening the course of the inflammation during the recovery period of experimental animals.

When interacting with cellular receptors of neutrophils, T- and B-lymphocytes, circulating immune complexes disrupt their functional activity. Strengthening the adhesion of neutrophils to the vascular endothelium is observed after phagocytosis of circulating immune complexes. As a result, they release a number of enzymes into the lumen of the bronchi, in particular elastase and proteinase, whose biological targets are cells of bronchopulmonary tissue [1, p. 995; 4, p. 125]. Together with these processes, changes in the structure and function of the epithelium of the bronchopulmonary apparatus begin to occur. They occur in response to inflammation reactions that progress due to prolonged exposure to proteinases and oxidants. The latter are expressed by effector cells of inflammation - neutrophils and macrophages. Goblet cells begin to actively produce and secrete mucous secrets, as a result of exposure to neutrophilic elastase [2, p. 1207; 3, p. 148].

The results of experimental studies allow us to draw certain conclusions. Nitrogen(IV) oxide during prolonged inhalation causes the formation of a local and systemic chronic inflammatory process in rats. Pathological processes that occur in response to exposure to nitrogen(IV) oxide are accompanied by changes both at the level of natural and adaptive immunity, and continue until the completion of the recovery period. The development and course of autoimmune processes in lung tissue can maintain the persistence of inflammation in the bronchopulmonary tract.

\section{References:}

1. Bagdonas, E., Raudoniute, J., Bruzauskaite, I., \& Aldonyte, R. (2015). Novel aspects of pathogenesis and regeneration mechanisms in COPD. International journal of chronic obstructive pulmonary disease, 10, 995.

2. Domej, W., Oettl, K., \& Renner, W. (2014). Oxidative stress and free radicals in COPDimplications and relevance for treatment. International journal of chronic obstructive pulmonary disease, 9, 1207.

3. Harkness, L. M., Kanabar, V., Sharma, H. S., Westergren-Thorsson, G., \& Larsson-Callerfelt, A. K. (2014). Pulmonary vascular changes in asthma and COPD. Pulmonary pharmacology \& therapeutics, 29(2), 144-155.

4. Ojo, O., Lagan, A. L., Rajendran, V., Spanjer, A., Chen, L., Sohal, S. S.,... \& Hackett, T. L. (2014). Pathological changes in the COPD lung mesenchyme-novel lessons learned from in vitro and in vivo studies. Pulmonary pharmacology \& therapeutics, 29(2), 121-128.

5. Groneberg, D. A., \& Chung, K. F. (2004). Models of chronic obstructive pulmonary disease. Respiratory research, 5(1), 18. 